\title{
Electro-optic steering of Nematicons
}

\author{
Gaetano Assanto, ${ }^{*}$ Armando Piccardi, Raouf Barboza, and Alessandro Alberucci \\ NooEL-Nonlinear Optics and OptoElectronics Lab, University Roma Tre 00146 Rome-Italy
}

Received November 28, 2011; accepted January 22, 2012; published March 31, 2012

\begin{abstract}
We study the angular steering of spatial solitons in nematic liquid crystals, i.e. nematicons, exploiting their electro-optic response. Approaches to electro-optic steering include acting on the optic axis and walk-off, propagating the soliton through a voltageadjusted refractive interface or getting it totally internally reflected. Using comb electrodes in a planar cell, maximum angular deflections of $70^{\circ}$ are expected with the standard nematic liquid crystal mixture E7.
\end{abstract}

One of the remarkable features of nematic liquid crystals (NLCs) is their sensitivity to electric fields, from static voltages to light. They exhibit large electro-optic and alloptical responses and significant tunability through molecular reorientation, i.e. the reorientation of the long molecular axes of constituent highly anisotropic (elongated) organic molecules [1]. Two-dimensional optical spatial solitons, i.e. self-confined beams with an invariant profile upon propagation, were demonstrated in an NLC via reorientation at the beginning of the century and have since stimulated sizable literature, because of both their fundamental interest in nonlinear optics and their potential applications in all-optical signal processing [2-12]. One of the most intriguing features of such solitons in NLCs, or nematicons, [12] is their capability of guiding and routing co-polarized signals: the ability to steer and/or bend a nematicon, in fact, corresponds to steering/routing the transmitted signals as well, realizing a completely reconfigurable scheme for spatial demultiplexing [3]. In this Letter we try to address the main avenues to angularly steer nematicons by way of applied voltage(s).

Nematicons are non-diffracting light beams which, excited in extraordinary polarization, are intense enough to "pull" the induced molecular dipoles towards the field vector, thereby increasing the extraordinary refractive index $n_{e}(\theta)$ as far as the angle $\theta$ between the dipoles and the wave vector remains below $\pi / 2$ :

$n_{e}(\theta)=\frac{n_{\perp} n_{\|}}{\sqrt{\left(n_{\|}^{2}-n_{\perp}^{2}\right) \cos ^{2} \theta+n_{\perp}^{2}}}$ with $n_{\|}$and $n_{\perp}$ the ordinary and extraordinary indices for field polarizations along and perpendicular to the long axis of the molecules, or the molecular director, respectively.

Since NLCs are typically birefringent positive uniaxials, an extraordinarily polarized beam propagating in the principal plane yz (see Fig. 1) will walk off the wave vector by an angle $\delta$, as large as $7^{\circ}$ in the mixture E7 in the NIR spectral range. One way to change the direction $\mathbf{S}$ of the beam is, therefore, to alter the walk-off angle by reorienting the optic axis (i.e. molecular director) of the NLC [13-15]. This can be accomplished by biasing the cell across the NLC thickness (along $\mathrm{x}$ ), in order to lift the director from the initial plane $y z$ and, by doing so, progressively reduce $\delta$ from 7 to nearly $0^{\circ}$. Fig. 2 shows the trajectories of nematicons launched $\| \mathbf{z}$ in E7 with the director initially at $\theta=\pi / 4$ with respect to $\mathbf{k} \| \mathbf{z}$ and for various applied voltages across the $100 \mu \mathrm{m}$ thickness. (a)

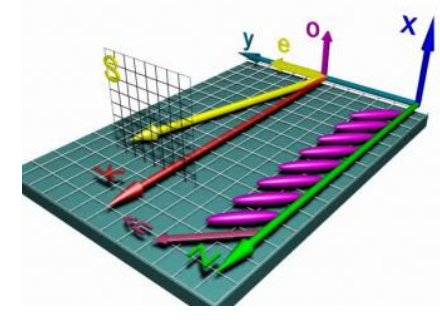

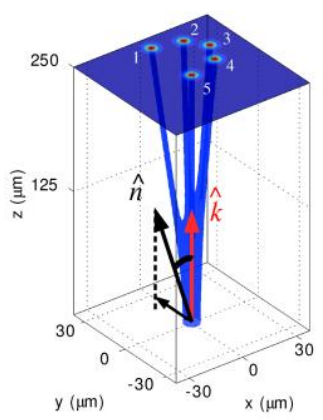

(b)
Fig. 1. (a) Initial orientation of an NLC in a planar cell, with the indication of an extraordinary polarized input field (e) in the principal plane yz, wave vector $(\mathbf{k})$ and Poynting vector $(\mathbf{S})$. The ellipses represent the long axes of the molecules. An applied voltage can lift them out of the plane until they align to $\mathbf{x}$. (b) 3D sketch of the Poynting vector of a nematicon launched with $\mathbf{k} \| \mathbf{z}$ for increasing voltage (from 1 to $5 \mathrm{~V}$ ) applied along $\mathbf{x}$.

*E-mail: assanto@uniroma3.it 


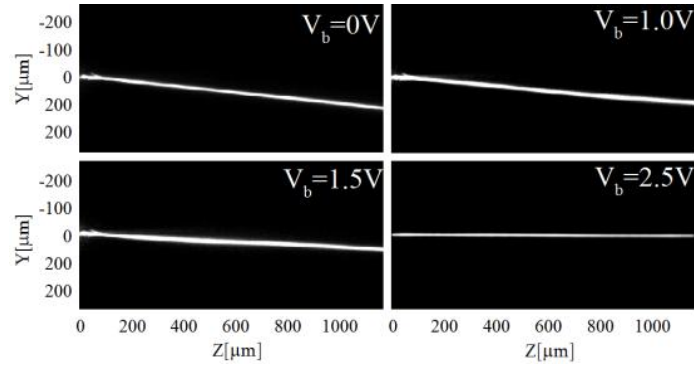

Fig. 2. Photographs of nematicons propagating in a planar NLC cell with E7, for various biases. The input beam was launched along $\mathbf{z}$ and formed a nematicon travelling at $\delta=7^{\circ}$ due to birefringence. Increasing the bias, the walk-off progressively reduced to nearly zero (last photo).

The previous approach, however quite effective, is limited by the amount of walk-off, about $7^{\circ}$ for a birefringence of $\sim 0.2$. Moreover, the beam is actually steered in 3D rather than in the input plane.

Refraction and/or total internal reflection (TIR) of a beam at an interface separating two dielectrics with a different refractive index can actually allow for deviations beyond the limit above. Since for nematicons the two dielectric regions need to be nematic liquid crystals, a simple approach to defining an interface is to use two (top) electrodes separated by a straight gap (parallel to the coordinate $p$ in Fig. 3(a)) and each connected to a voltage supply, in order to apply two different biases $\mathrm{V}_{1}$ and $\mathrm{V}_{2}$ to the two NLC regions (see Fig. 3(a)) [16-18]. Depending on the angle of incidence $\Phi_{\mathrm{I}}$, the input beam in Region 1 will form a nematicon able to refract (at an angle $\Phi_{R}$ ) from Region 1 to Region 2 or to keep propagating after TIR in Region 1 at $\Phi_{\mathrm{TIR}}$, as sketched in Fig. 3(b).

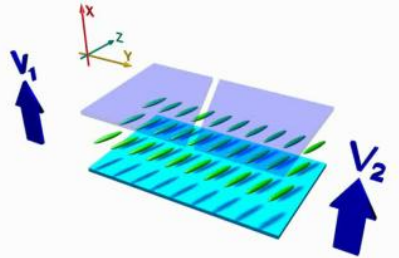

(a)

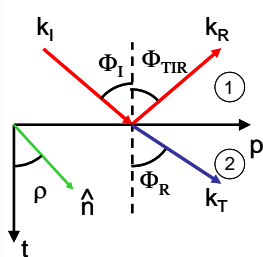

(b)
Fig. 3. NLC cell with a straight inter-electrode gap $(\| p)$ defining a graded interface between two NLC dielectric regions separately biased by $V_{1}$ and $V_{2}$. (a) Sketch of the cell; (b) impinging and outgoing rays.

Sample experimental results at $\lambda=1.064 \mu \mathrm{m}$ in a planar cell with E7 are displayed in Fig. 4 for various bias mismatches $\mathrm{V}_{1}-\mathrm{V}_{2}$. Fig. 4(a) is the photograph/top view of a nematicon going straight through the interface (indicated by a dashed line) as $\mathrm{V}_{1}=\mathrm{V}_{2}$ (i.e. no interface). Fig. 4(b) shows a nematicon refracted as $V_{1}<V_{2}$ (i.e. Region 1 rarer than Region 2), with a deviation of $18^{\circ}$. Fig. 4(c) shows a nematicon undergoing total internal reflection as $V_{1}>V_{2}$ (i. e. Region 1 denser than Region
2 ), with a deviation of $22^{\circ}$. This approach to steering was, therefore, able to yield overall deviations as large as $40^{\circ}$ using moderate voltages in a planar $100 \mu \mathrm{m}$ thick cell.
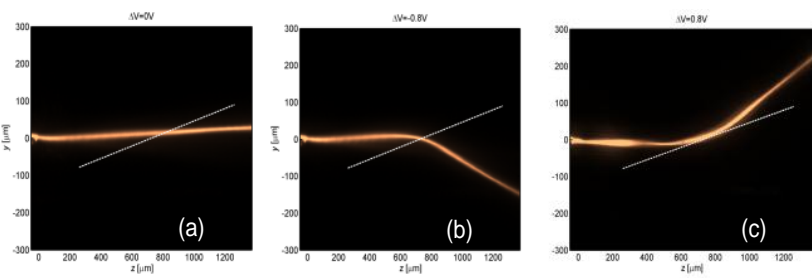

Fig. 4. Observation of near infrared nematicon (a) going straight through the interface; (b) nematicon refraction; (c) nematicon total internal reflection.

A bias-defined interface, however, not only alters the refractive index, but also modifies the orientation of the optic axis in each region, thereby incurring in out-of-plane steering, i.e. one of the drawbacks indicated with reference to the first method.

Extra flexibility for electro-optic steering of a selftrapped beam is offered by the use of interdigitated electrodes, as sketched in Fig. 5.

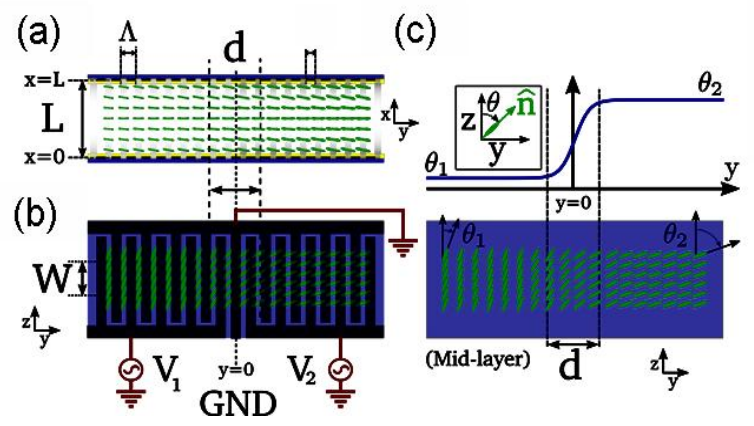

Fig. 5. (a) Side view and (b) top view of a planar NLC cell designed with interdigitated electrodes on both top and bottom interfaces. (c) Distribution of the optic axis in the cell mid-plane (bottom) and corresponding orientation $\theta$ versus y (top).

Resorting to a double set of comb-patterned electrodes in-plane nematicon propagation and steering can be achieved by voltage-tuning the effective anisotropy. The NLC layer is confined between two glass plates parallel to $\mathrm{yz}$; at each interface, two sets of transparent electrodes with fingers along $y$, period $\Lambda$ and 50:50 mark-to-space ratio permit the application of the bias. Each electrode pair has a ground terminal in $\mathrm{y}=0$ : this way a voltage $\mathrm{V}_{1}$ $\left(\mathrm{V}_{2}\right)$ on the left (right) comb can twist the molecular dipoles and the optic axis in yz throughout the NLC thickness in the region underneath (Fig. 5(c)) [19]. 


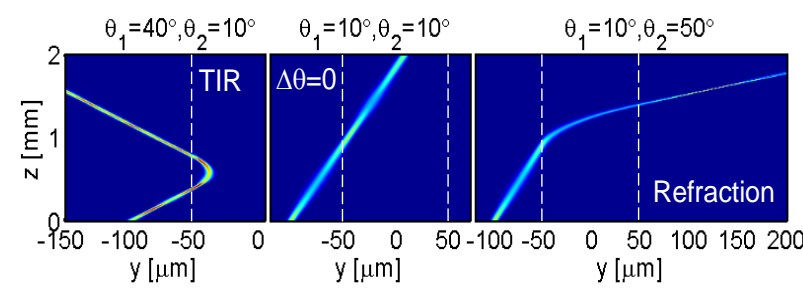

Fig. 6. (a) Calculated evolution of a $3 \mathrm{~mW}$ nematicon for various electro-optic orientations in the two regions (left and right of the $\mathrm{d}=100 \mu \mathrm{m}$ thick graded interface); the dashed white lines indicate the interface borders; the dotted lines indicate the trajectory for $\theta_{0}=10^{\circ}$ (with no biases).

For $\Lambda$ much smaller than the thickness, the applied electric field is mainly along $\mathrm{y}$, being the $\mathrm{x}$-component of the electric field cut off by nonlocality, thus the director rotates in the plane yz and keeps the beam in the plane. By adjusting the orientation $\theta$ in each region, this configuration allows the nematicon steering by refraction and TIR, with overall deflections as large as $40^{\circ}$ for input beams incident with a wave vector along $\mathrm{z}$ and a graded interface of thickness $\mathrm{d} \approx 100 \mu \mathrm{m}$.

Even larger deflections can be achieved by injecting the beam with an incidence angle slightly below the critical angle. For a beam of $5 \mu \mathrm{m}$ waist and $1.064 \mu \mathrm{m}$ wavelength, the maximum obtainable deflection of $70^{\circ}$ corresponds to the incidence at nearly $25^{\circ}$ with respect to $\mathrm{z}$ and careful tuning of both voltages, taking also into account the birefringent walk-off.

Figure 7 shows such maximum achievable deflections indicating changes both in the wave vector and the Poynting vector.

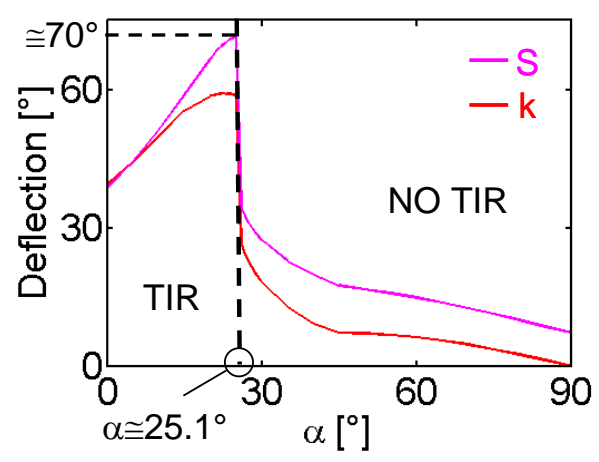

Fig. 7. Maximum angular deflection of wave vector $\mathbf{k}$ and Poynting vector $\mathbf{S}$ versus incidence angle $\alpha$ with respect to $\mathrm{y}$.
Numerical results and geometric optics predictions are in good agreement. Noteworthy, these calculated angular deviations of self-confined beams, to be achieved electrooptically by applying voltage biases of only a few Volts, rely on a single dielectric interface and could be further increased by engineering multiple (cascaded) interfaces.

In conclusion, nematicons can be efficiently readdressed exploiting the NLC high birefringence and the associated strong electro-optic effect. Electric control on nematicon trajectory can be based upon walk-off variations and graded index interfaces, the latter ensuring deflection angles as large as $70^{\circ}$ in an NLC with a birefringence equal to 0.2 . The phenomena illustrated in this Letter can find application in the design and realization of reconfigurable all-optical communications networks.

Acknowledgements: Early work on nematicon steering by voltage was carried out by $M$. Peccianti in collaboration with C. Conti, C. Umeton, A. De Luca, M. Kaczmarek and A. Dyadyusha.

\section{References}

[1] I. C. Khoo, Phys. Rep. 471, 221 (2009).

[2] M. Peccianti, G. Assanto, A. De Luca, C. Umeton, I.C. Khoo, Appl. Phys. Lett. 77, 7 (2000).

[3] M. Peccianti, and G. Assanto, Opt. Lett. 26, 1690 (2001).

[4] M. Peccianti, and G. Assanto, Phys. Rev. E Rapid Commun. 65 , 035603 (2002).

[5] M. Peccianti, K.A. Brzdąkiewicz, and G. Assanto, Opt. Lett. 27, 1460 (2002).

[6] M. Peccianti, G. Assanto, A. De Luca, C. Umeton, M. A. Karpierz, and I.C. Khoo, J. Commun. Technol. Electron. 47, 790 (2002).

[7] G. Assanto and M. Peccianti, IEEE J. Quantum Electron. 39, 13 (2003).

[8] G. Assanto, M. Peccianti, C. Conti, Acta Phys. Pol. A 103, 16 (2003).

[9] M. Peccianti, C. Conti, G. Assanto, A. De Luca, C. Umeton, J. Nonl. Opt. Phys. Mat. 12, 525 (2003).

[10] G. Assanto, C. Conti and M. Peccianti, Int. Mod. Phys. Lett. B 18, 2819 (2004).

[11] G. Assanto and M. Peccianti, Mol. Cryst. Liq. Cryst. 488, 163 (2008).

[12] G. Assanto, M. Karpierz, Liq. Cryst. 36, 1161 (2009).

[13] M. Peccianti, C. Conti, G. Assanto, A. De Luca, and C. Umeton, Nature 432, 733 (2004).

[14] A. Alberucci, M. Peccianti, G. Assanto, G. Coschignano, A. De Luca, and C. Umeton, Opt. Lett. 30, 1381 (2005).

[15] G. Assanto, C. Umeton, M. Peccianti, and A. Alberucci, J. Nonl. Opt. Phys. Mat. 15, 33 (2006).

[16] M. Peccianti, A. Dyadyusha, M. Kaczmarek, and G. Assanto, Nat. Phys. 2, 737 (2006).

[17] M. Peccianti, G. Assanto, A. Dyadyusha, and M. Kaczmarek, Phys. Rev. Lett. 98, 113902 (2007).

[18] G. Assanto, and M. Peccianti, J. Nonl. Opt. Phys. Mat. 16, 37 (2007).

[19] R. Barboza, A. Alberucci, and G. Assanto, Opt. Lett. 36, 2611 (2011). 